\title{
Study of emission mechanism of GRBs probed by the gamma-ray polarization with IKAROS-GAP
}

\author{
Daisuke Yonetoku ${ }^{1}$, Toshio Murakami ${ }^{1}$, Tomonori Sakashita ${ }^{1}$, \\ Yoshiyuki Morihara ${ }^{1}$, Shuichi Gunji ${ }^{2}$, Tatehiro Mihara ${ }^{3}$, \\ Kenji Toma ${ }^{4}$, and GAP team \\ ${ }^{1}$ College of Science and Engineering, School of Mathematics and Physics, Kanazawa \\ University, Kakuma, Kanazawa, Ishikawa 920-1192, Japan, \\ email: (DY) yonetoku@astro.s.kanazawa-u.ac.jp \\ ${ }^{2}$ Department of Physics, Faculty of Science, Yamagata University, 1-4-12, Koshirakawa, \\ Yamagata, Yamagata 990-8560, Japan \\ ${ }^{3}$ Cosmic Radiation Laboratory, RIKEN, 2-1, Hirosawa, Wako City, Saitama 351-0198, Japan \\ ${ }^{4}$ Department of Earth and Space Science, Osaka University, Toyonaka 560-0043, Japan
}

\begin{abstract}
We report a polarization measurement in prompt $\gamma$-ray emission of GRB 100826A with the Gamma-Ray Burst Polarimeter (GAP) aboard the small solar power sail demonstrator IKAROS. We detected a firm change of polarization angle (PA) during the prompt emission with $99.9 \%(3.5 \sigma)$ confidence level, and an average polarization degree $(\Pi)$ of $27 \pm 11 \%$ with $99.4 \%(2.9 \sigma)$ confidence level. Here the quoted errors are given at $1 \sigma$ confidence level for two parameters of interest. Non-axisymmetric (e.g., patchy) structures of the magnetic fields and/or brightness inside the relativistic jet are therefore required within the observable angular scale of $\sim \Gamma^{-1}$. Our observation strongly indicates that the polarization measurement is a powerful tool to constrain the GRB production mechanism, and more theoretical works are needed to discuss the data in more details.
\end{abstract}

Keywords. Gamma-Ray Bursts, polarization, emission mechanism

\section{Introduction}

Gamma-ray bursts (GRBs) are the most energetic explosions in the universe. Since the discovery of X-ray afterglow of GRBs by BeppoSAX Costa et al. (1997) and the identification of optical counterparts at cosmological distance, many observational facts and theoretical works have led us to understand the nature of GRBs. However, a crucial issue still remaining to answer is how to release such a huge energy as $\gamma$-ray photons. In spite of extensive discussions with the spectral and lightcurve information being collected, there are still several possible models of GRBs Mészáros (2006). The polarimetric observations provide us completely different information. A firm detection of linear polarization of $\gamma$ ray photons will further constrain the emission models Lazzati (2006), Toma et al. (2009).

\section{Data Analyses}

The GAP detected GRB 100826A on 26 August 2010 at 22:57:20.8 (UT). The lightcurve of the prompt emission is shown in Fig. 1 (left). First of all, we performed the polarization analysis for the entire dataset, but we set only an upper limit of $\Pi<30 \%$ ( $2 \sigma$ confidence level). Therefore we divided the entire data into two time intervals, Interval- 1 and -2 , as labeled in the lightcurve in Figure 1 (right). (Although Interval-2 has several spikes, we 

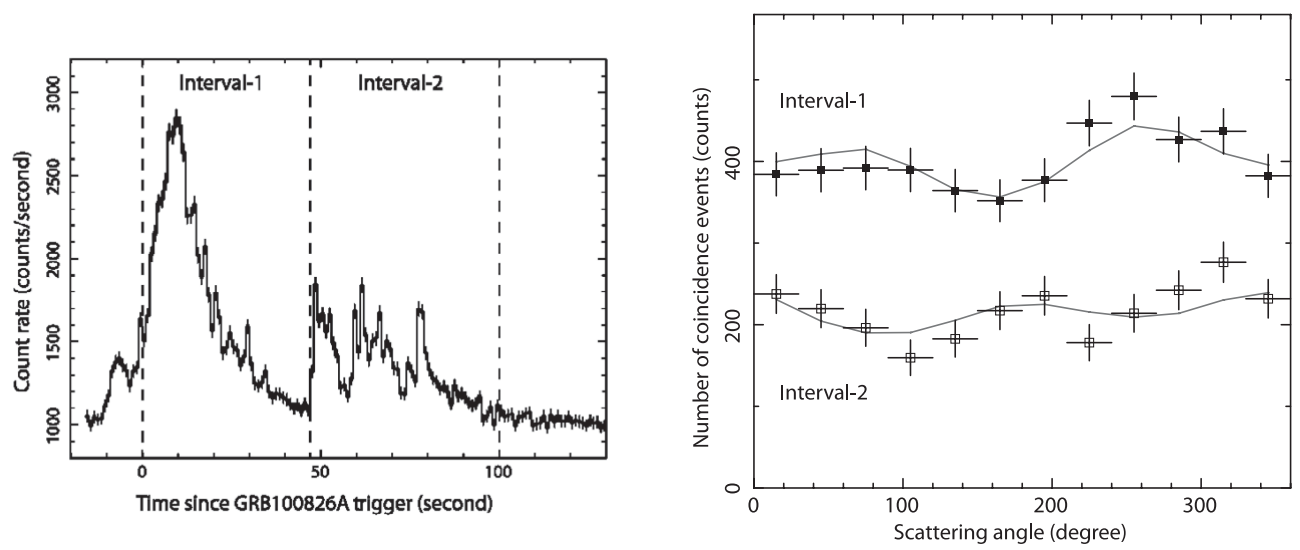

Figure 1. (Left) The lightcurve of GRB 100826A. (Right) The modulation curve of each interval shown in the right panel.

combined all of them to keep photon statistics.) We investigated the polarization degrees, $\Pi$, and the polarization angle $\left(\phi_{p}\right)$ separately for Interval-1 and -2 .

The observed modulation curve is shown in Fig. 1 (right). The response of GAP for irradiation from 20.0 degree off-axis is modeled by the Monte-Carlo method with Geant 4 simulator. The gray solid lines in Fig. 1 (right) are the best-fit functions for Interval-1 and -2 . The best values are $\Pi_{1}=25 \pm 15 \%$ with $\phi_{p 1}=159 \pm 18$ degrees for Interval-1 and $\Pi_{2}=31 \pm 21 \%$ with $\phi_{p 2}=75 \pm 20$ degrees for Interval-2, respectively. Hereafter the quoted errors are $1 \sigma$ confidence for two parameters of interest. The significance of polarization detection is rather low: $95.4 \%$ and $89.0 \%$ for Interval-1 and -2 , while the difference of polarization angles is significant with $99.9 \%(3.5 \sigma)$ level.

In the next step, we performed a combined fit for the two intervals, assuming that the polarization degree for Interval-2 is the same as that for Interval-1. This means that we treat $\Pi$ as one free parameter to improve the statistics with the reduction of model parameters. Here the two polarization angles were still free parameters for both intervals, because a change of angle is apparent. The best-fit polarization degree is $\Pi=27 \pm 11 \%$ with $\chi^{2}=21.8$ for 19 degrees of freedom. The significance of detection of polarization is $99.4 \%(2.9 \sigma)$ confidence level.

We detected significant polarization from two more events, GRB 110301A and GRB 110721A. Detailed discussion can be found in Yonetoku et al. (2011b). We consider that the emission mechanism of GRBs may be synchrotron radiation in relatively coherent magnetic fields, and the existence of patchy emission regions can explain the observed rapid change of polarization angle.

\section{References}

Costa, E. et al., 1997, Nature, 387, 783-785

Lazzati, D. et al., 2006, New Journal of Physics, 8, 131

Mészáros, P. 2006, Rep. Prog. Phys., 69, 2259

Toma, K., Sakamoto, T., Zhang, B., et al. 2009, ApJ, 698, 1042

Yonetoku, D., Murakami, T., Gunji, S., Mihara, T., et al., 2011, PASJ, 63, 3

Yonetoku, D., Murakami, T., Gunji, S., Mihara, T., Toma, K., et al., 2011, ApJ, 743, L30 